\title{
NEWAGE: direction-sensitive direct dark matter search
}

\section{Kentaro Miuchi ${ }^{*} a$, Ryota Yakabe $a$, Takashi Hashimoto $a$, Tomonori Ikeda $a$, Ryosuke Taishaku $a$, Miki Nakazawa $a$, Yushiro Yamaguchi $a$, Tomohiro Inada $a$, Kiseki Nakamura $b$, Toru Tanimori $b$, Hidetoshi Kubo $b$, Atsushi Takada $b$, Hiroyuki Sekiya $c$, Atsushi Takedac}

aDepartment of Physics, Kobe University, Rokkodai, Nada-ku Kobe-shi, Hyogo, 657-8501, Japan

bDepartment of Physics, Kyoto University, Oiwakecho, Sakyo-ku Kyoto-shi, Kyoto, 606-8502,

Japan

c Kamioka Observatory, ICRR, The University of Tokyo, Gifu, 506-1205 Japan

E-mail: miuchiephys.sci.kobe-u.ac.jp

\begin{abstract}
NEWAGE is a direction-sensitive direct dark matter search experiment. NEWAGE uses a micro time-projection chamber with a detection volume of $31 \times 31 \times 41 \mathrm{~cm}^{3}$, which is read by a macro-petterned gaseous detectors, $\mu$-PIC. Underground measurements with the current detector, NEWAGE-0.3b ', have been performed since 2013. After the first direction-sensitive limits drived from 31.6 live-days of NEWAGE-0.3b' data were published, measurements were continued and the preliminary results from an addtional 170 days of results are reported here. In order to improve the sensitivity, low $\alpha$-particle emission $\mu$-PICs are being developed. Studies on negative-ion gases are also on-going. These recent activities that aim to improve the sensitivity are also presented here.
\end{abstract}

38th International Conference on High Energy Physics

3-10 August 2016

Chicago, USA

${ }^{*}$ Speaker. 


\section{Introduction}

One quarter of the universe is thought to be in a form of an unidentified elementary particle, or dark matter[四]. Weakly interacting massive particles(WIMPs) are a well-motivated dark matter candidate. The detection of nuclear recoil tracks is thought to be capable of providing a strong evidence of dark matter detection[]. Among the various methods of nuclear track detection, gaseous detectors are the most widely studied for application in direction-sensitive dark matter searches. The NEWAGE experiment adopted micro-patterned gaseous detectors(MPGDs) in the directional dark matter search for the first time.

\section{NEWAGE}

An MPGD-based direction-sensitive dark matter search, NEWAGE, was proposed with realistic experimental demonstrations in 2004[[3], NEWAGE provided the first directional dark matter search results in 2007[䧃]. NEWAGE has been taking data in Kamioka Observatory underground laboratory since 2007 and has since updated the directional limits [[] [ [6].

A picture of the currently running NEWAGE detector, NEWAGE-0.3b', is shown in Fig. $\mathbb{m}$. The detector chamber is visible in the center while the electronics system with field-programmable gate arrays(FPGAs) is shown on the left. The gas circulation system with cooled charcoal is shown to the right of the chamber. The interior of the detector chamber and the micro time-projectionchamber ( $\mu$-TPC) are shown in Fig. $\square$. The $\mu$-TPC consists of a two-dimensional fine-pitch imaging device known as a micro-pixel chamber ( $\mu$-PIC), a gas electron multiplier (GEM), and a detection volume $\left(31 \times 31 \times 41 \mathrm{~cm}^{3}\right)$ filled with $\mathrm{CF}_{4}$ gas at $0.1 \mathrm{~atm}$. Details of the detector system and its performance are described in Ref.[目].

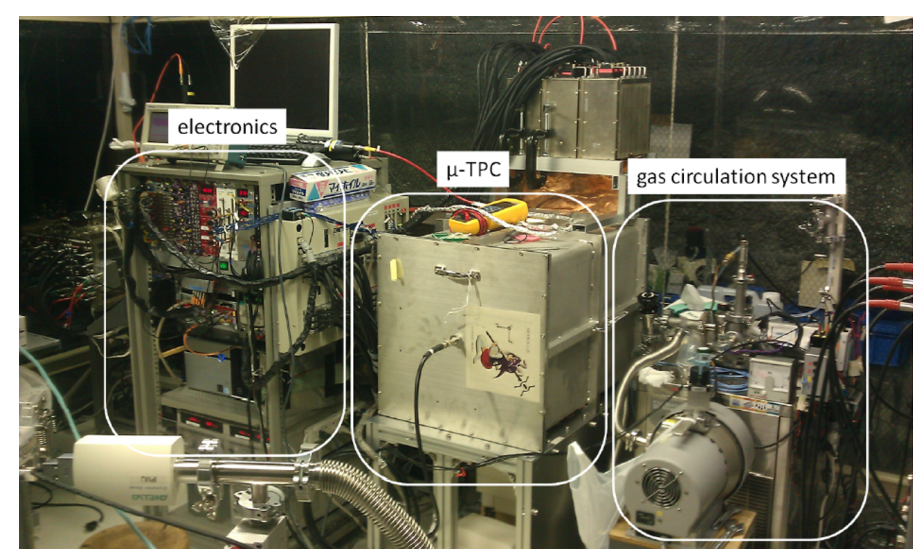

Figure 1: Picture of the NEWAGE-0.3b' system. From the left: the electronics system, the $\mu-$ TPC chamber, and the gas circulation system.

\section{Underground Measurements}

The first direction-sensitive limits obtained with NEWAGE-0.3b were reported in 2015[6]. In addition to this 31.6 live-day measurement, we have run the detector for another 172.08 live-days. 


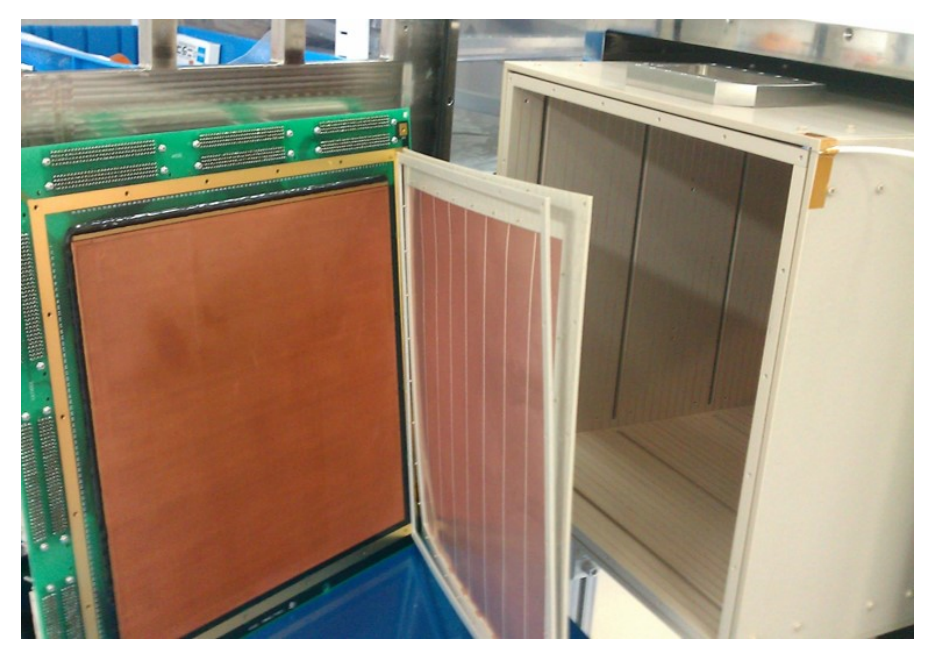

Figure 2: Interior view of the NEWAGE-0.3b' $\mu$-TPC. From the left: the $\mu$-PIC, the GEM, and the field cage.

Together with a statistical increase by a factor of seven, we have also updated the event selections. The new spectrum is shown in Fig. [1. The event rate at the threshold decreased by a factor of more than two and the statistical error has been reduced. We are now conducting directional analysis, and the new limits will be reported in the future work.

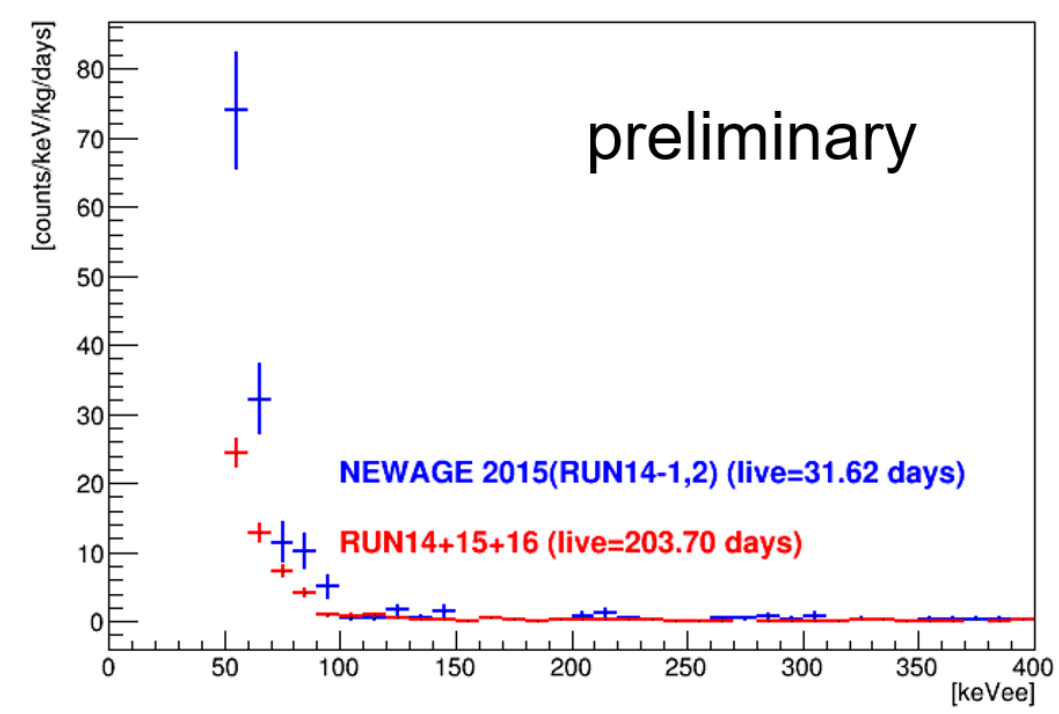

Figure 3: Preliminary energy spectrum of the RUN 14+15+16 measurement(red). The energy spectrum from our previous publication is shown for comparison with blue the histogram[ 6$]$.

\section{Recent R \& D Activities}

\subsection{Low $\alpha \mu$-PIC development}

From the study on the background in the NEWAGE 2015 data, the dominant background 
source was found to be alpha particles from ${ }^{238} \mathrm{U}$ and ${ }^{232} \mathrm{Th}$ contamination in the $\mu$-PIC. The materials of the $\mu$-PIC were investigated with germanium detectors and most of these radioactive isotopes were found to be contaminated in the glass cloth (GC) used to reinforce the polyimide (PI) insulator. The schematic cross-sectional drawings of this "standard" $\mu$-PIC are shown in the left panel of Fig. $⿴$. There are three layers of polyimide reinforced with glass cloth (PI(w/GC)). Alpha particles from the top layer were found to be the the major source of the low energy background.

We decided to develop a new type of $\mu$-PIC, namely low $\alpha \mu$-PIC, by replacing the top layer of $\mathrm{PI}(\mathrm{w} / \mathrm{GC})$ with less radioactive material. A new material comprising of a $5 \mu \mathrm{m}$ thick PI layer without glass cloth and $75 \mu \mathrm{m}$ epoxy glue was used for the development process. This material was found to contain about two orders of magnitude less ${ }^{238} \mathrm{U}\left(<3.0 \times 10^{-9} \mathrm{~g} / \mathrm{g}\right)$ and ${ }^{232} \mathrm{Th}(<$ $6.8 \times 10^{-9} \mathrm{~g} / \mathrm{g}$ ). A cross section of this low- $\alpha \mu$-PIC is shown in the right panel of Fig. 田. It was decided that the $800 \mu \mathrm{m}$ PI (w/GC) should continue to be used because a) $\alpha$ particles from this layer do not reach the surface and b) fabrication risk is reduced with minimal changes in the materials. The largest "risk" involved in changing the materials is the alignment of layers with different coefficients of thermal expansion.

Low- $\alpha \mu$-PICs with a detection area of $10 \times 10 \mathrm{~cm}^{2}$ were successfully created by Dai Nippon Printing Co., Ltd. A picture of the $10 \times 10 \mathrm{~cm}^{2}$ low $\alpha \mu$-PIC is shown in the left panel of Fig. 1 . In the right panel of Fig. I, a microscopic view is shown. It can be observed that the anode electrodes are placed in the center of the cathode electrodes. This means that the "risk" of misalignment was controlled well during the fabrication process.

\subsection{Detector tests with negative ion gas}

The other way to reduce the background from the $\mu$-PIC is to fiducialize the absolute drift length " $z$ " of the event position. This fiducialization was thought to be difficult for self-triggering TPCs used for dark matter searches. Recently, the DRIFT group found a way to realize the "zfiducialization", first with $\mathrm{CS}_{2}$ gas and then with $\mathrm{SF}_{6}$ gas [[]]. In these gases, more than two species of negative ions with different drift speeds are produced and the absolute drift distance can be measured using the difference in the drift times of the ions. We have begun testing $\mathrm{SF}_{6}$ gas with our system and have measured a sufficient gas gain of about 2000[प]].

\subsection{Future Prospects}

Low- $\alpha \mu$-PICs with a detection area of $31 \times 31 \mathrm{~cm}^{2}$ are being developed. One of these low$\alpha \mu$-PICs will be installed in NEWAGE-0.3b' in 2017. This will reduce the background by two orders of magnitude. ASICs for the readout of the negative ion gas TPC are also being developed. These ASICs have longer shaping time compared to those used with electron drifting TPCs because of the slower drift velocity. With these electronics, we will be able to reduce the background not only from the $\mu$-PIC but also from the drift plane. By cutting 3 times of the spatial resolution, we expect a background reduction of another two orders of magnitude. We are designing a larger detector with a detection volume of $\sim 1 \mathrm{~m}^{3}$ with a $50 \mathrm{~cm}$ polyethylene neutron shield. With these improvement, we are planing to cover the DAMA's spin-dependent allowed region and further in a few years. 

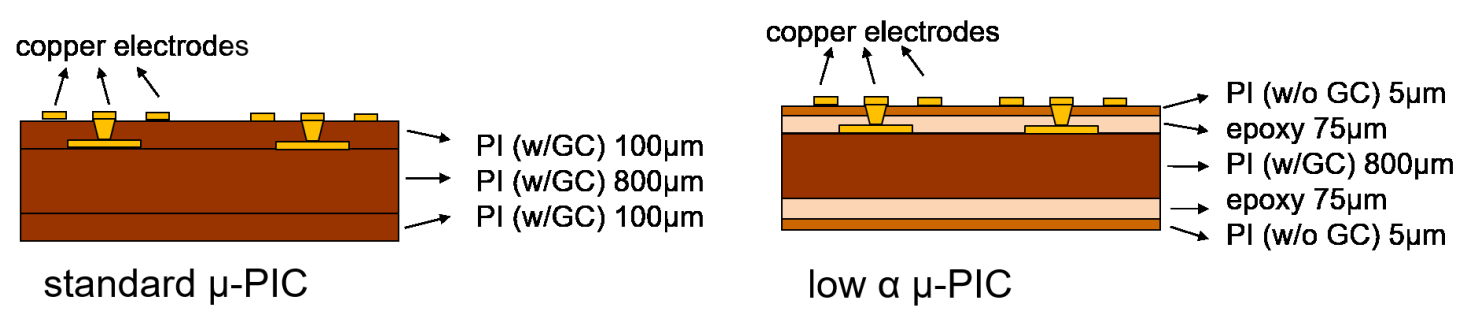

Figure 4: Cross sections of the standard $\mu$-PIC(left) and the low- $\alpha \mu$-PIC(right).

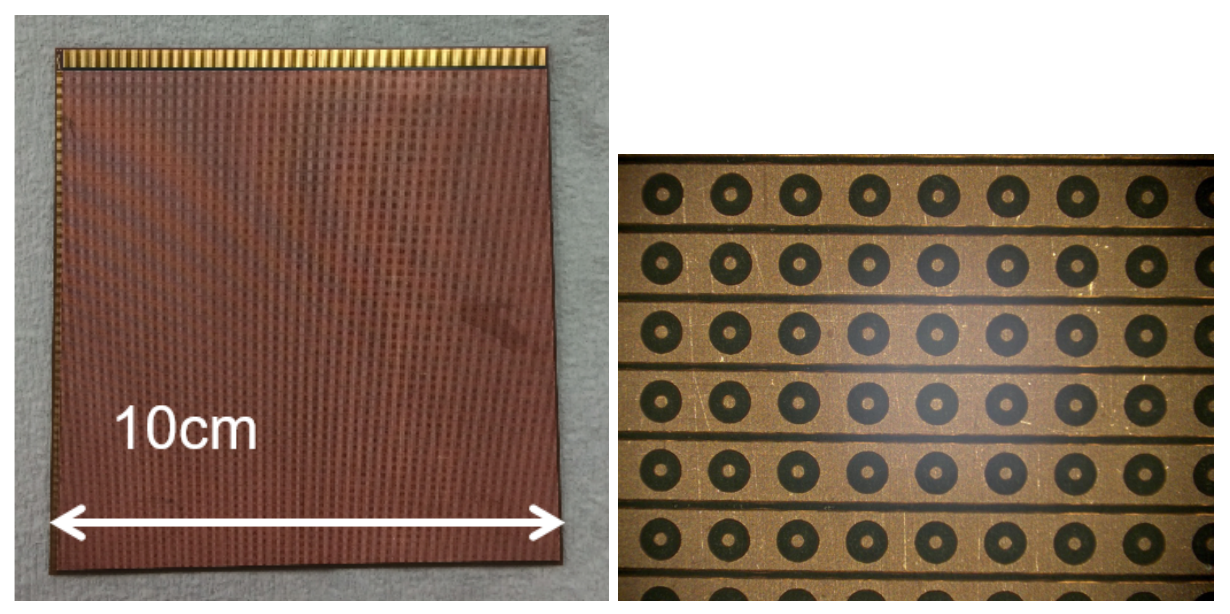

Figure 5: Picture of the low- $\alpha 10 \times 10 \mathrm{~cm}^{2} \mu$-PIC(left). Microscopic view is shown in the right panel.

\section{Acknowledgments}

This study was supported by KAKENHI Grants-in-Aid (19684005, 23684014, 26104005, and 16H02189) and Program for Advancing Strategic International Networks to Accelerate the Circulation of Talented Researches, JSPS, Japan(R2607)

\section{References}

[1] Planck Collaboration, A\&A 571 (2014) A16

[2] D.N. Spergel, Phys. Rev. D 37 (1988) 1353.

[3] T. Tanimori et al., Phys. Lett. B 578 (2004) 241

[4] Kentaro Miuchi et al., Phys. Lett. B 654 (2007) 58

[5] Kentaro Miuchi et al., Phys. Lett. B 686 (2010) 11

[6] K. Nakamura et al., Prog. Theor. Exp. Phys. 14 (2015) 043F01.

[7] R. Yakabe et al., JPS Conf. Proc. Proceedings of International Symposium on Radiation Detectors and Their Uses (ISRD2016) 11 (2016) 040002.

[8] J.B.R. Battatet al. Physics of the Dark Universe 9-10 (2015) 1.

[9] Nguyen Phan, oral presentation at CYGNUS 2015 ,Los Angeles, June 2015. 
[10] T. Ikeda et al., Proceedings. of 4th International Conference on Micro Pattern Gaseous Detectors (MPGD 2015) in press 